\title{
A Recursive Frequency Smoothing Method of Cyclic Spectrum Density Estimation
}

\author{
Jianzhong Dai \\ National University of Defense Technology \\ Xuzhe Feng ( $\nabla$ kd805fxz@163.com ) \\ National University of Defense Technology \\ Jianyun Chen \\ National University of Defense Technology \\ Shuhua Yan \\ National University of Defense Technology \\ Aiai Jia \\ National University of Defense Technology
}

\section{Research}

Keywords: Cyclic spectrum density estimation, Frequency smoothing, Computational cost, Recursive method

Posted Date: May 18th, 2020

DOl: https://doi.org/10.21203/rs.3.rs-28727/v1

License: (c) (i) This work is licensed under a Creative Commons Attribution 4.0 International License. Read Full License 


\section{A Recursive Frequency Smoothing Method of Cyclic}

\section{Spectrum Density Estimation}

Jianzhong Dai, Xuzhe Feng *, Jianyun Chen, Shuhua Yan and Aiai Jia

College of Intelligent Science and Technology, National University of Defense Technology,

Changsha 410073, China;

* Correspondence: kd805fxz@163.com; Tel.: +86-137-5516-7689

Abstract: Cyclic spectrum density estimation plays a significant role in cyclostationary signals analysis. Generally, the methods of cyclic spectrum density estimation are mainly focus in time-domain, such as Fast fourier transform Accumulation Method (FAM) and Strip Spectral Correlation Algorithm (SSCA). In this paper, based on the principle of frequency smoothing methods, an improved method in frequency-domain was proposed, which could reduce the computational cost greatly by using recursion. The recursive method was based on the fact that the range difference of smoothing window was small when window slid. In other words, we could get a new point of cyclic spectrum density just by computing the complex conjugate products of newly coming into window. In addition, the simulation results were given at last, which showed the good performance of our proposed method. method

\section{Introduction}

Many cyclostationary signals are widely used in engineering fields, such as communications, telemetry, radar and sonar. Traditionally, these signals are treated as non-stationary. However, it is more suitable to be modeled as cyclostationary because their statistics are periodic due to modulating, sampling and other signal processing operations. As a consequence, the analysis of cyclostationary get a lot of attention in signal processing and it is widely used in many fields, including signal reconnaissance, modulation recognition and sonar detection ${ }^{[1-4]}$. In the analysis of cyclostationary signal, the cyclic 
spectrum density estimation plays an important role for its calculation cost and implementation diversity.

29 Generally, there are two types of algorithms to estimate cyclic spectrum density. One is time smoothing 30 algorithms, such as Fast fourier transform Accumulation Method (FAM) and Strip Spectral Correlation 31 Algorithm (SSCA). The other one is frequency smoothing algorithms, such as Frequency Smoothing 32 Method (FSM) [5-7].

The basic estimation methods in time smoothing algorithms and frequency smoothing algorithms are both given in Ref. [5]. The performance and computational efficiency of the commonly used estimation methods are analyzed and compared explicitly in Ref. [6]. In addition, a conclusion is drawn that the computational cost of frequency smoothing algorithms is greater than that of time smoothing algorithms ${ }^{[6]}$. As a consequence, almost all the estimation methods in succedent studies are focus in time smoothing algorithms, and the frequency smoothing algorithms are ignored actually.

In recent years, the study of cyclic spectrum density mainly focuses on these fields: characteristic of cyclic spectrum density, optimization of implementation and application of cyclic spectrum density. Cyclostationary Generalized Frequency Division Multiplexing (GFDM) signal properties in flexible cognitive radio is analyzed in Ref. [8]. The algorithms to calculate cyclic spectrum density is optimized in Refs. [9-12], while all these algorithms are based on time smoothing method. Cyclic spectrum density can be used to detect signal feature ${ }^{[13]}$ and signal classification ${ }^{[14]}$. With regards to implementation, time smoothing algorithms generally employ a coarse-grain channelizer, where the bandwidth of each channel is a large fraction of the analysis bandwidth. On the other hand, frequency smoothing algorithms generally employ a fine-grain channelizer, where the bandwidth of each channel is a small fraction of the analysis bandwidth [15]. That is to say, if a higher frequency resolution is required under the same analysis bandwidth, the performance of frequency smoothing algorithms is better. Therefore, it is necessary to study an improved frequency smoothing algorithm by reducing its computational cost.

The primary objective of this paper is to introduce a recursive approach method for calculating cyclic spectrum density based on frequency smoothing method, and compare the computational cost and performance with other algorithms. The proposed recursive frequency smoothing algorithms has reduced computational cost successfully, what's more, it has good performances in detecting cyclic frequency at different Signal to Noise Ratio (SNR) levels. is described. Section 3 presents implementation and improvement of frequency smoothing method. 
58 Section 4 analyses and compares different algorithms in computational cost and performance. Section 5

59 shows some simulation results by using the proposed recursive frequency smoothing method and SSCA.

60 At last, the paper ends with the conclusions provided by Section 6.

\section{Definition of Cyclic Spectrum Density}

Suppose $x(t)$ is a non-stationary complex signal with zero mean, the time-vary autocorrelation of $x(t)$ can be defined as [16]

$$
R_{x}(t, \tau)=E\left\{x(t) x^{*}(t-\tau)\right\},
$$

64 , the autocorrelation function can also be written as

$$
R_{x}(t, \tau)=\lim _{N \rightarrow \infty} \frac{1}{2 N+1} \sum_{n=-N}^{N} x\left(t+n T_{0}\right) x^{*}\left(t+n T_{0}-\tau\right)
$$
where $R_{x}(t, \tau)$ is a periodic function with a period of $T_{0}$. When $N$ tends to infinity, Eq. (2) is equivalent to Eq. (1). It can also be expanded into a Fourier series

$$
R_{x}(t, \tau)=\sum_{m=-\infty}^{\infty} R_{x}^{\alpha}(\tau) e^{+j \frac{2 \pi}{T_{0}} m t}=\sum_{m=-\infty}^{\infty} R_{x}^{\alpha}(\tau) e^{+j 2 \pi \alpha t},
$$

70 where $\alpha=\frac{m}{T_{0}}$ and $j=\sqrt{-1}$. The Fourier coefficients are given by

$$
R_{x}^{\alpha}(\tau)=\frac{1}{T_{0}} \int_{-T_{0} / 2}^{T_{0} / 2} R_{x}(t, \tau) e^{-j 2 \pi \alpha t} d t
$$

Substitute Eq. (2) into Eq. (4), and let $T=(2 N+1) T_{0}$, we can obtain,

$$
R_{x}^{\alpha}(\tau)=\lim _{T \rightarrow \infty} \frac{1}{T} \int_{-T / 2}^{T / 2} x(t) x^{*}(t-\tau) e^{-j 2 \pi \alpha t} d t=<x(t) x^{*}(t-\tau) e^{-j 2 \pi \alpha t}>_{t},
$$
a function of $\tau$. 
Whereas in practice, a symmetrical form is more common used

$$
y_{\tau}(t)=x(t+\tau / 2) x^{*}(t-\tau / 2)
$$

So, the cyclic autocorrelation with symmetrical form can be written as

$$
R_{x}^{\alpha}(\tau)=\left\langle x(t+\tau / 2) x^{*}(t-\tau / 2) e^{-j 2 \pi \alpha t}\right\rangle_{t}
$$
say, it can be got from the Fourier transform of cyclic autocorrelation $R_{x}^{\alpha}(\tau)$

$$
S_{x}^{\alpha}(f)=\int_{-\infty}^{\infty} R_{x}^{\alpha}(\tau) e^{-j 2 \pi f \tau} d \tau
$$

78

and set

$$
\left\{\begin{array}{c}
u(t)=x(t) e^{-j \pi \alpha t} \\
v(t)=x(t) e^{j \pi \alpha t},
\end{array}\right.
$$

83 the cyclic autocorrelation in Eq. (9) can be expressed as the cross-correlation of $u(t)$ and $v(t)$.

$$
\begin{aligned}
R_{x}^{\alpha}(\tau) & =R_{u v}(\tau)=\left\langle u(t+\tau / 2) v^{*}(t-\tau / 2)\right\rangle_{t} \\
& =\lim _{T \rightarrow \infty} \frac{1}{T} \int_{-T / 2}^{T / 2} u(t+\tau / 2) v^{*}(t-\tau / 2) d t
\end{aligned},
$$

$$
S_{x}^{\alpha}(f)=U(f) V^{*}(f),
$$

where $U(f)$ and $V(f)$ are the Fourier transform of $u(t)$ and $v(t)$, respectively. $U(f)=X(f+\alpha / 2)$ and $V(f)=X(f-\alpha / 2)$, and $X(f)$ is the Fourier transform of $x(t)$. 
In engineering calculation, the average $T$ in Eq. (11) is impossible of being infinite. Therefore,

89 the issue of cyclic spectrum density estimation must be studied at the condition of the limited $T$. If the center time is $t$, the Fourier transform of the signal $x(t)$ is

$$
X_{T}(t, f)=\int_{t-T / 2}^{t+T / 2} x(u) e^{-j 2 \pi f u} d u,
$$

91

The time-varied spectrum of $U(f)$ and $V(f)$ can be acquired by shifting frequency $\pm \alpha / 2$ of

92 $X_{T}(t, f)$,

$$
\left\{\begin{array}{l}
U_{T}(t, f)=X_{T}(t, f+\alpha / 2) \\
V_{T}(t, f)=X_{T}(t, f-\alpha / 2)
\end{array},\right.
$$

$$
S_{u v, T}(t, f)_{\Delta t}=\frac{1}{\Delta t} \int_{-\Delta t / 2}^{\Delta t / 2} \frac{1}{T} U_{T}(t, f) V_{T}^{*}(t, f) d t,
$$

$$
\begin{aligned}
S_{x}^{\alpha}(f) & =S_{u v}(f)=\lim _{\Delta t \rightarrow \infty} \lim _{T \rightarrow \infty} S_{u v, T}(t, f)_{\Delta t} \\
& =\lim _{\Delta t \rightarrow \infty} \lim _{T \rightarrow \infty} \frac{1}{\Delta t} \int_{-\Delta t / 2}^{\Delta t / 2} \frac{1}{T} U_{T}(t, f) V_{T}^{*}(t, f) d t
\end{aligned},
$$

$$
S_{x}^{\alpha}(f)=\lim _{\Delta t \rightarrow \infty \Delta f \rightarrow 0} \lim _{\Delta t} \frac{1}{\Delta t} \int_{t-\Delta t / 2}^{t+\Delta t / 2} \Delta f U_{1 / \Delta f}(u, f) V_{1 / \Delta f}^{*}(u, f) d u,
$$

$$
\begin{gathered}
\left\{\begin{array}{l}
U_{1 / \Delta f}(t, f)=X_{1 / \Delta f}(t, f+\alpha / 2) \\
V_{1 / \Delta f}(t, f)=X_{1 / \Delta f}(t, f-\alpha / 2)
\end{array},\right. \\
X_{1 / \Delta f}(t, f)=\int_{t-1 / 2 \Delta f}^{t+1 / \Delta f} x(u) e^{-j 2 \pi f u} d u,
\end{gathered}
$$




$$
\begin{gathered}
S_{x}^{\alpha}(f)=\lim _{\Delta f \rightarrow 0} \lim _{\Delta t \rightarrow \infty} \frac{1}{\Delta f} \int_{f-\Delta f / 2}^{f+\Delta f / 2} \frac{1}{\Delta t} U_{\Delta t}(t, v) V_{\Delta t}^{*}(t, v) d v, \\
\left\{\begin{array}{l}
U_{\Delta t}(t, f)=X_{\Delta t}(t, f+\alpha / 2) \\
V_{\Delta t}(t, f)=X_{\Delta t}(t, f-\alpha / 2)
\end{array},\right. \\
X_{\Delta t}(t, f)=\int_{t-\Delta t / 2}^{t+\Delta t / 2} x(u) e^{-j 2 \pi f u} d u,
\end{gathered}
$$

In Ref. [1], it has proved that Eq. (17) and Eq. (22) is equivalent when $\Delta t \Delta f \square 1$.

101 is given as follows [1]

$$
\begin{gathered}
S_{x_{\Delta t}}^{\alpha}(f)_{\Delta f}=\frac{1}{M} \sum_{\gamma=-(M-1) / 2}^{(M-1) / 2} \frac{1}{N} X_{\Delta t}\left(t, f+\frac{\alpha}{2}+\gamma F_{s}\right) X_{\Delta t}^{*}\left(t, f-\frac{\alpha}{2}+\gamma F_{s}\right), \\
X_{\Delta t}(t, f)=\sum_{k=0}^{N-1} a_{r}\left(k T_{s}\right) x\left(t-k T_{s}\right) e^{-j 2 \pi f\left(t-k T_{s}\right)}
\end{gathered}
$$

102 where $a_{r}(n)$ is data window function, $M$ is the number of frequency smoothing range (number of

103 frequency samples in rectangle window), $N$ is the number of time samples, $F_{s}=1 / N T_{s}$ is the

104 frequency sample increment, $T_{s}$ is the time sampling increment, $\Delta f=M F_{s}$ is the width of rectangle smoothing window in frequency-domain, and $\Delta t$ is length of time series and $\alpha=2 m F_{s}$ is the cyclic

106 frequency. The algorithm architecture is depicted in Figure 1. In this algorithm, $f=(p+l) /(2 N)$ and $\alpha=(p-l) / N$, where $p$ is a certain frequency of $X_{\Delta t}(t, f)$ and $l$ is a certain frequency of

$108 X_{\Delta t}{ }^{*}(t, f)$. For simplicity, the treatment in this paper is restricted to cyclic spectrum density analysis of 109 a single real-valued signal.

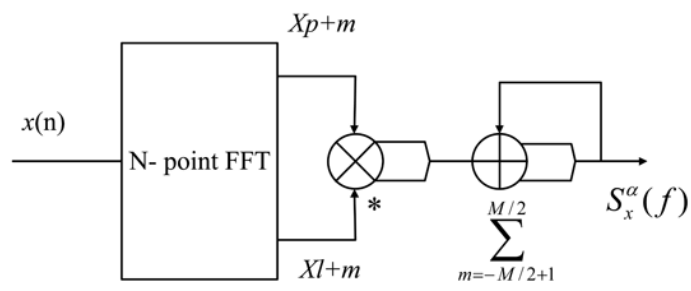


113 products of Fourier transform of $x(t)$ for fixed spectrum frequency $f_{0}$ and cycle frequency $\alpha_{0}$.

114 Since $S_{x}^{\alpha}(f)$ is the function of both cyclic frequency $\alpha$ and spectrum frequency $f$, the two

115 variables can change respectively for acquiring estimation of $S_{x}^{\alpha}(f)$. As depicted in Figure 2, the fixed

116 cycle frequency $\alpha_{0}$ is the distance between two smoothing windows, $f_{0}$ and $f_{0}+F_{s}$ are the centers

117 of window before and after window movement, respectively, where $F_{s}$ is the increment of spectrum

118 frequency sample.

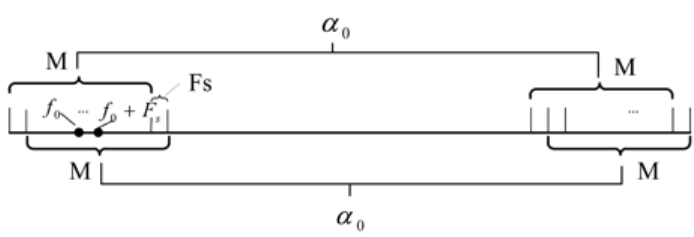

Figure 2. Sketch of smoothing window move-fixed $\alpha$

When $f$ is fixed, $\alpha_{0}$ and $\alpha_{0}+2 F_{s}$ are the distances of the center of window before and after window movement, respectively, where $2 F_{s}$ is the increment of cyclic frequency samples. The sketch of smoothing window is depicted in Figure 3.

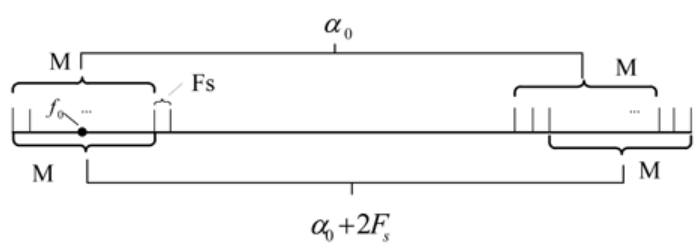

Figure 3. Sketch of smoothing window move-fixed $f$

Although the range is changed after the window movement, the conjugated multiply relationship between each pair of spectrums is not changed if they still located in the window. Moreover, the content in window is changed very small before and after the movement. That is to say, the difference between the successive cyclic correlations is small. Based on this principle, the computational cost can be reduced effectively. The detailed deduce procedure is shown below.

According to Eq. (23), $S_{x_{\Delta t}}^{\alpha}(f)_{\Delta f}$ is a function of two variables with $\alpha$ and $f$, where these two variables are both in frequency domain. The increment of spectrum frequency and cyclic frequency are 
134 fixed and $f$ move with $F_{s}$, we can obtain

$$
S_{x_{\Delta t}}^{\alpha}\left(f_{0}+F_{s}\right)_{\Delta f}=\frac{1}{M} \sum_{\gamma=-(M-1) / 2}^{(M-1) / 2} \frac{1}{N} X_{\Delta t}\left(f_{0}+F_{s}+\frac{\alpha}{2}+\gamma F_{s}\right) X_{\Delta t}{ }^{*}\left(f_{0}+F_{s}-\frac{\alpha}{2}+\gamma F_{s}\right),
$$

$$
\begin{aligned}
S_{x_{\Delta t}}^{\alpha}\left(f_{0}+F_{s}\right)_{\Delta f}= & \frac{1}{M} \sum_{p=-(M-1) / 2+1}^{(M-1) / 2+1} \frac{1}{N} X_{\Delta t}\left(f_{0}+\frac{\alpha}{2}+p F_{s}\right) X_{\Delta t}^{*}\left(f_{0}-\frac{\alpha}{2}+p F_{s}\right) \\
= & \frac{1}{M} \sum_{p=-(M-1) / 2}^{(M-1) / 2} \frac{1}{N} X_{\Delta t}\left(f_{0}+\frac{\alpha}{2}+p F_{s}\right) X_{\Delta t}^{*}\left(f_{0}-\frac{\alpha}{2}+p F_{s}\right) \\
& +\frac{1}{M N}\left[X_{\Delta t}\left(f_{0}+\frac{\alpha}{2}+\frac{M+1}{2} F_{s}\right) X_{\Delta t}^{*}\left(f_{0}-\frac{\alpha}{2}+\frac{1+M}{2} F_{s}\right)-X_{\Delta t}\left(f_{0}+\frac{\alpha}{2}+\left(-\frac{M-1}{2}\right) F_{s}\right) X_{\Delta t}^{*}\left(f_{0}-\frac{\alpha}{2}+\left(-\frac{M-1}{2}\right) F_{s}\right)\right] \\
& =S_{x_{\Delta}}^{\alpha}\left(f_{0}\right)_{\Delta f}+\frac{1}{M N}\left[X_{\Delta t}\left(f_{0}+\frac{\alpha}{2}+\frac{M+1}{2} F_{s}\right) X_{\Delta t}^{*}\left(f_{0}-\frac{\alpha}{2}+\frac{M+1}{2} F_{s}\right)-X_{\Delta t}\left(f_{0}+\frac{\alpha}{2}+\frac{1-M}{2} F_{s}\right) X_{\Delta t}^{*}\left(f_{0}-\frac{\alpha}{2}+\frac{1-M}{2} F_{s}\right)\right]
\end{aligned}
$$

$$
\begin{aligned}
& S_{x_{\Delta t}}^{\alpha}\left(f_{0}+F_{s}\right)_{\Delta f}-S_{x_{\Delta t}}^{\alpha}\left(f_{0}\right)_{\Delta f} \\
= & \frac{1}{M N}\left[X_{\Delta t}\left(f_{0}+\frac{\alpha}{2}+\frac{M+1}{2} F_{s}\right) X_{\Delta t}^{*}\left(f_{0}-\frac{\alpha}{2}+\frac{M+1}{2} F_{s}\right)-X_{\Delta t}\left(f_{0}+\frac{\alpha}{2}+\frac{1-M}{2} F_{s}\right) X_{\Delta t}^{*}\left(f_{0}-\frac{\alpha}{2}+\frac{1-M}{2} F_{s}\right)\right]
\end{aligned}
$$

When $\alpha$ is fixed, due to the window movement, the difference between two cyclic spectrum density points is equal to the difference between the conjugated products of the points that just come into and go out window. As a result of iterative, we just need to compute the difference of two conjugated products instead of $M$ complex conjugated products for acquiring the cyclic spectrum density in a new point. Furthermore, if the memory is sufficient, only one conjugated complex product which comes into the smoothing window justly is needed to be computed. $S_{x_{\Delta t}}^{\alpha}(-f)_{\Delta f}=S_{x_{\Delta t}}^{\alpha}(f)_{\Delta f}$ and $S_{x_{\Delta t}}^{-\alpha}(f)_{\Delta f}=S_{x_{\Delta t}}^{\alpha *}(f)_{\Delta f}$. Then only one quadrant values need to be

145 computed, and the other quadrant values can be acquired by shifting. What's more, according to the

146 above iterative, the initial value is different when $\alpha$ is different. For a fixed $\alpha$, the initial value is the

147 accumulation of $M$ complex conjugated products when the distance of window center is $\alpha_{0}$ and $f=0$, as shown in Eq. (28) 


$$
S_{x_{\Delta t}}^{\alpha_{0}}(0)_{\Delta f}=\frac{1}{M} \sum_{\gamma=-(M-1) / 2}^{(M-1) / 2} \frac{1}{N} X_{\Delta t}\left(\frac{\alpha_{0}}{2}+\gamma F_{s}\right) X_{\Delta t}^{*}\left(-\frac{\alpha_{0}}{2}+\gamma F_{s}\right),
$$

Through the above improvement works, the computational cost of FSM can be reduced greatly.

\section{Analysis and Comparison of Different Algorithms}

Some algorithms of cyclic spectrum density estimation are compared in this section. $N^{\prime}=P / 4$. $2 N \log _{2} N$

$174 \quad M$ is the width of frequency smoothing window, and is $N / 16$ in usual. 
Recursion....................... $4\left(\frac{N}{2}-\frac{M}{2}\right)\left(\frac{N}{2}-\frac{M}{2}\right)$

178 Figure 4. It can be observed that computational cost of FSM is the most, FAM is the second, and SSCA

179 is the least. This conclusion agrees with that of Ref. [6]. However, the computational cost of recursive FSM is reduced greatly, and is better than that of SSCA.

Table 1. Computational cost of different algorithms.

\begin{tabular}{cc}
\hline Algorithms & Computational cost \\
\hline FAM & $6 P N^{\prime}+2 P N^{\prime} \log _{2} N^{\prime}+4 P N^{\prime 2}+2 P N^{\prime 2} \log _{2} P$ \\
SSCA & $6 P N^{\prime}+2 P N^{\prime} \log _{2} N^{\prime}+4 N^{\prime 2}+2 N N^{\prime} \log _{2} N$ \\
FSM & $2 N \log _{2} N+8 M\left(\frac{N}{2}-\frac{M}{2}\right)\left(\frac{N}{2}-\frac{M}{2}\right)$
\end{tabular}

Recursive

FSM

$$
2 N \log _{2} N+4\left(\frac{N}{2}-\frac{M}{2}\right)\left(\frac{N}{2}-\frac{M}{2}\right)
$$

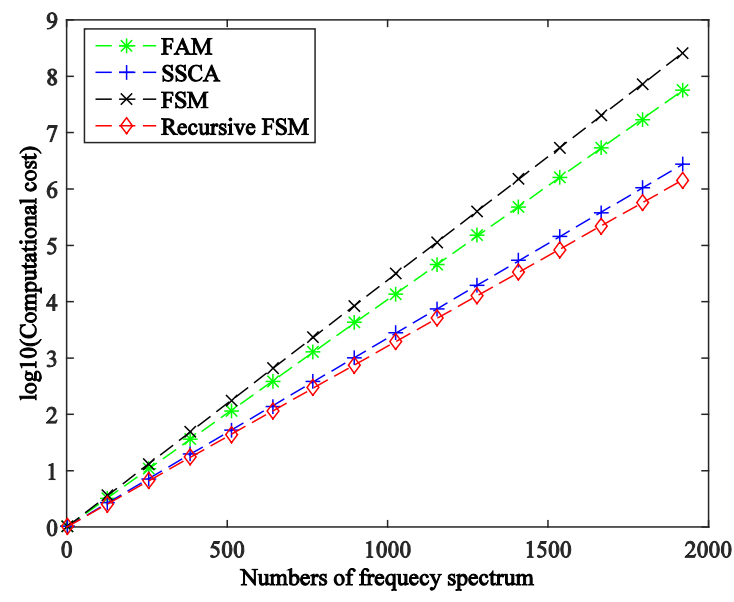

$$
x(t)=\cos \left(2 \pi f_{c} t+\phi(t)+\phi_{0}\right)=b(t) \cos \left(2 \pi f_{c} t+\phi_{0}\right),
$$


where $b(t)=\sum_{n=-\infty}^{\infty} b_{n} q\left(t-n T_{0}-t_{0}\right)$, and $b_{n}$ is a random sequence whose value is \pm 1 (corresponding to the phase 0 and $\pi$ ), and $q(t)$ is rectangular pulse. Its theoretical cyclic spectrum density is

$$
S_{B P S K}^{\alpha}(f)=\left\{\begin{array}{c}
\frac{1}{4 T_{0}}\left[Q\left(f+f_{c}+\frac{\alpha}{2}\right) Q\left(f+f_{c}-\frac{\alpha}{2}\right)+Q\left(f-f_{c}+\frac{\alpha}{2}\right) Q\left(f-f_{c}-\frac{\alpha}{2}\right)\right] \exp \left(-j 2 \pi \alpha t_{0}\right), \alpha=\frac{p}{T_{0}} \\
\frac{1}{4 T_{0}}\left[Q\left(f+\frac{p}{2 T_{0}}\right) Q\left(f_{c}-\frac{p}{2 T_{0}}\right)\right] \exp \left(-j 2 \pi t_{0} \frac{p}{T_{0}} \pm 2 \phi_{0}\right), \alpha=\frac{p}{T_{0}} \pm 2 f_{c}
\end{array},\right.
$$
That is to say, the cyclic spectrum density has a peak at $\alpha=\frac{p}{T_{0}}$ or $\alpha=\frac{p}{T_{0}} \pm 2 f_{c}, p$ is an integer, and $Q(f)$ is the Fourier transform of $q(t) . \alpha$ contains information about carrier frequency and code rate, and can be used in BPSK modulation recognition.

In order to evaluate the performance of the proposed recursive FSM, simulations are carried out in 194 certain conditions. Assume parameters of the signal as follows: BPSK modulation, sample frequency $f_{s}=1200 \mathrm{kHz}$, carrier frequency $f_{c}=150 \mathrm{kHz}$, code rate $f_{0}=7500 \mathrm{~Hz}$, white Gaussian noise and calculation points $N=1024$. The cyclic spectrum densities calculated by SSCA and recursive FSM at different SNR levels are shown in Figure 5-7. According to Eq. (29), cyclic frequency $\alpha$ can take a section of $S_{B P S K}^{\alpha}(f)$ for $f=0$, which can be used in signal detection and modulation recognition. The cyclic frequency $\alpha$ calculated by SSCA and recursive FSM respectively at different SNR levels are shown in Figure 8-10.
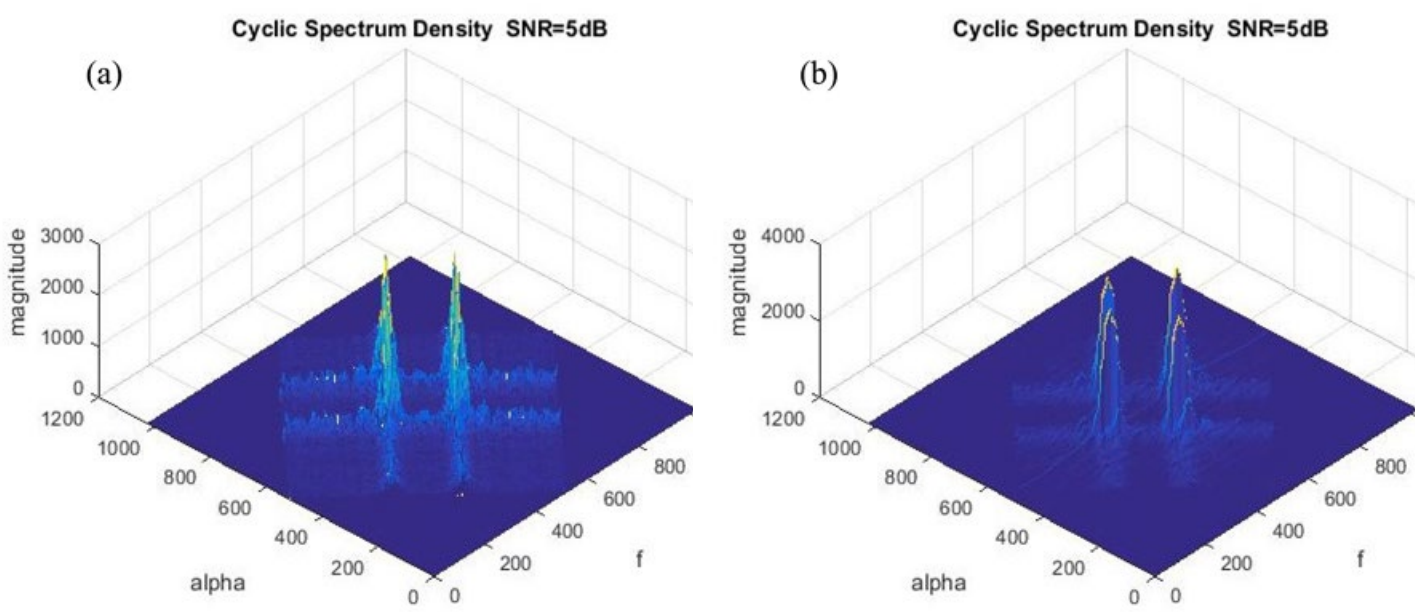

Figure 5. SNR $=5 \mathrm{~dB}$. Cyclic spectrum density calculated by (a) SSCA and (b) recursive FSM. 
(a)

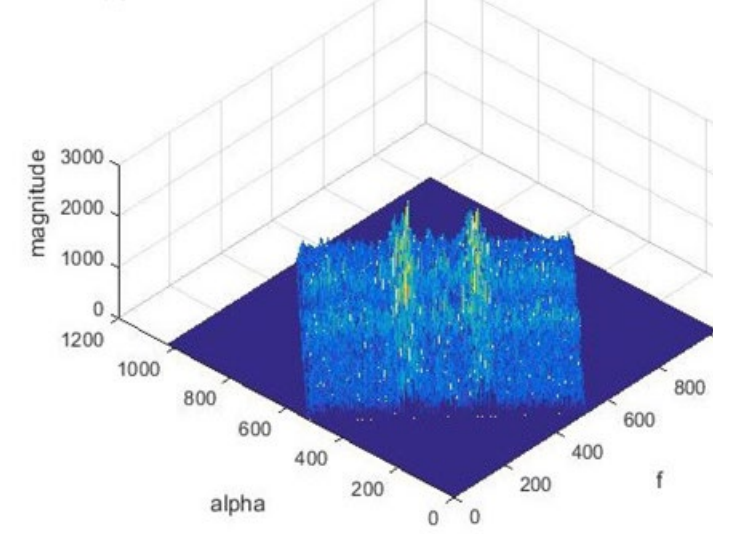

(b)

Cyclic Spectrum Density SNR=0dB

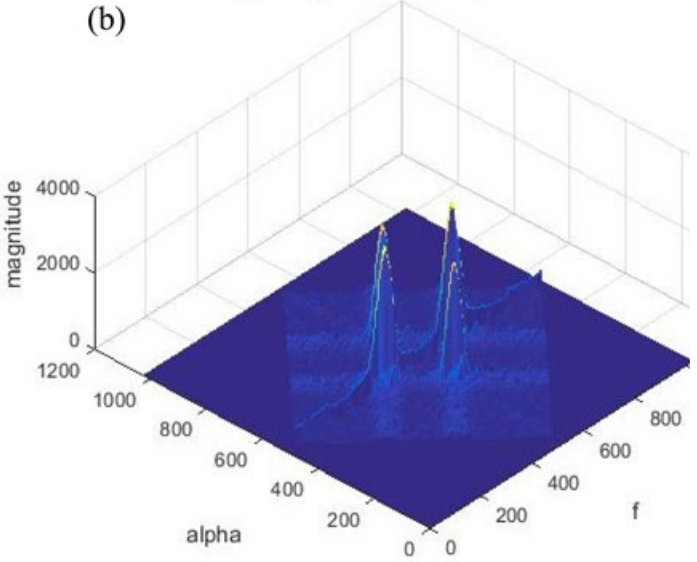

Figure 6. $\mathrm{SNR}=0 \mathrm{~dB}$. Cyclic spectrum density calculated by (a) SSCA and (b) recursive FSM.
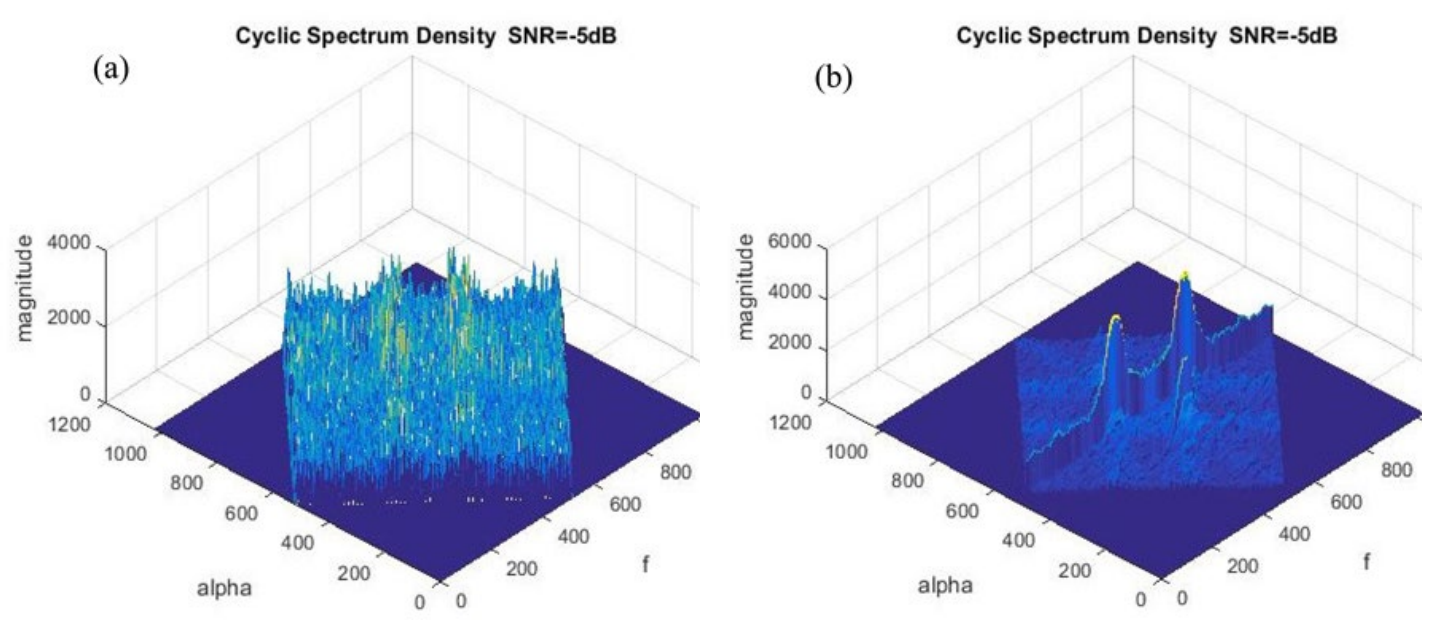

Figure 7. SNR $=-5 \mathrm{~dB}$. Cyclic spectrum density calculated by (a) SSCA and (b) recursive FSM.
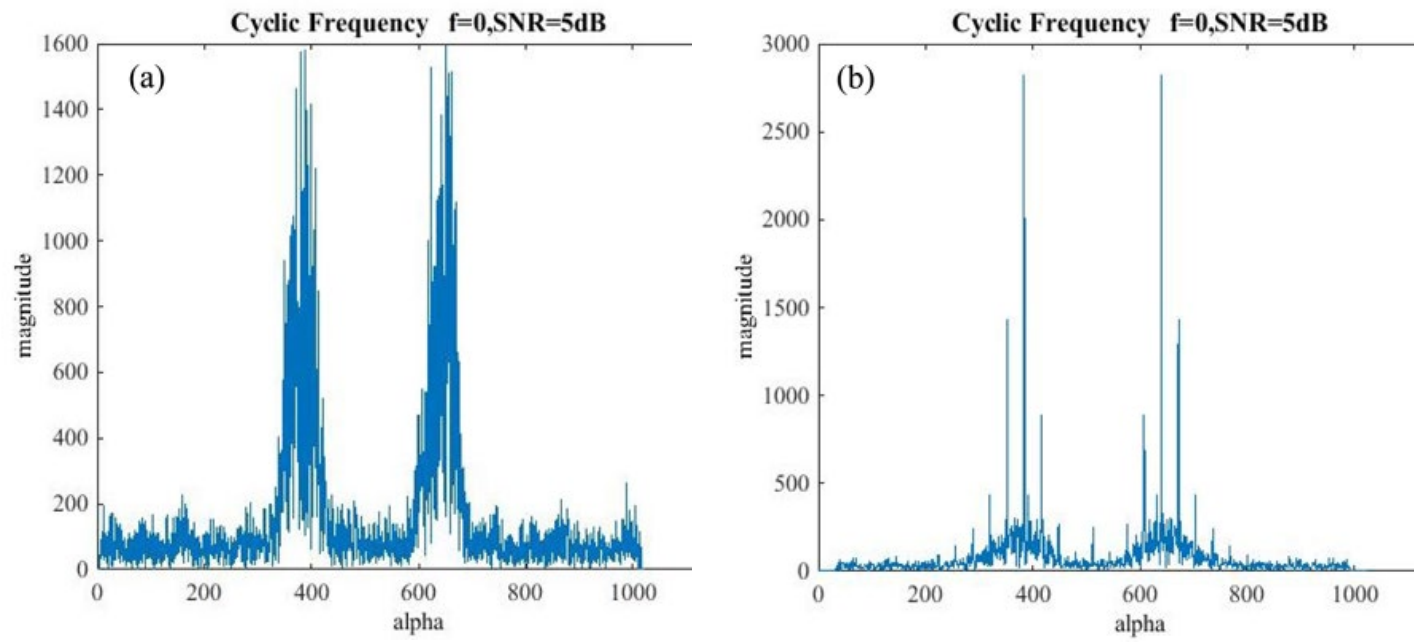

Figure 8. $\mathrm{SNR}=5 \mathrm{~dB}$. The cyclic frequency $\alpha$ calculated by (a) SSCA and (b) recursive FSM. 

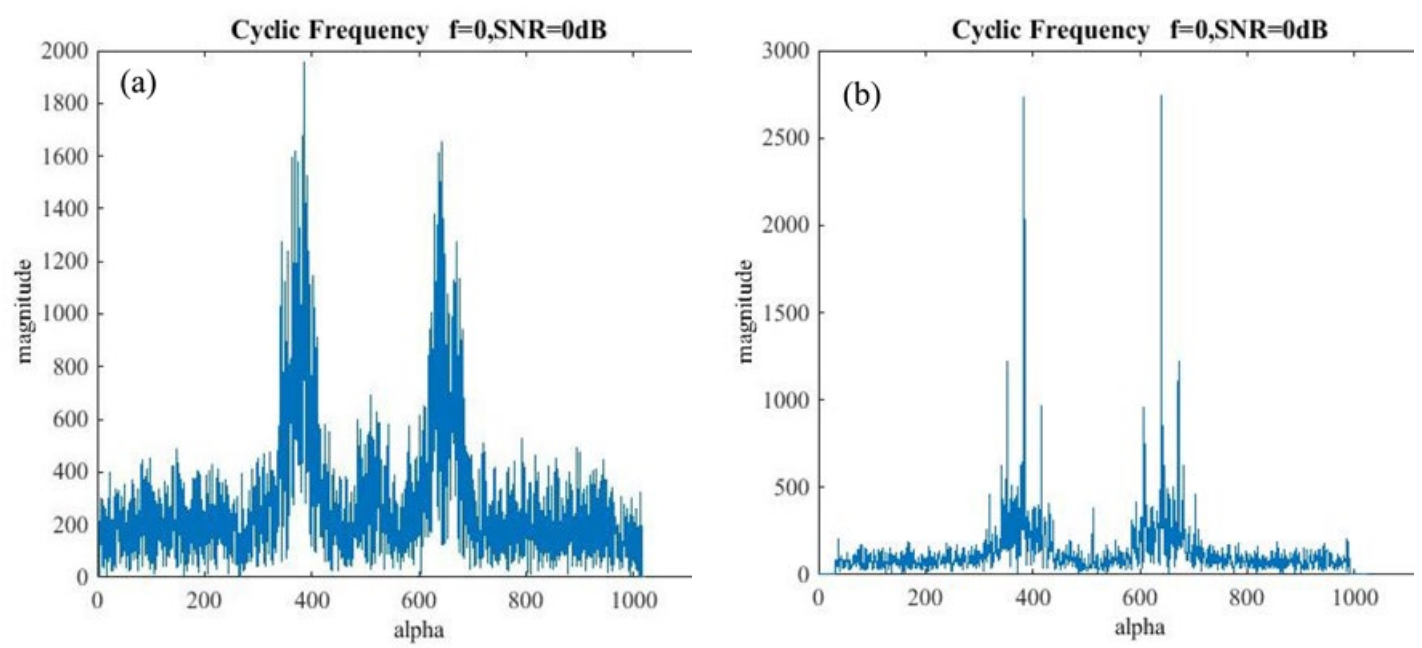

Figure 9. $\mathrm{SNR}=0 \mathrm{~dB}$. The cyclic frequency $\alpha$ calculated by (a) SSCA and (b) recursive FSM.
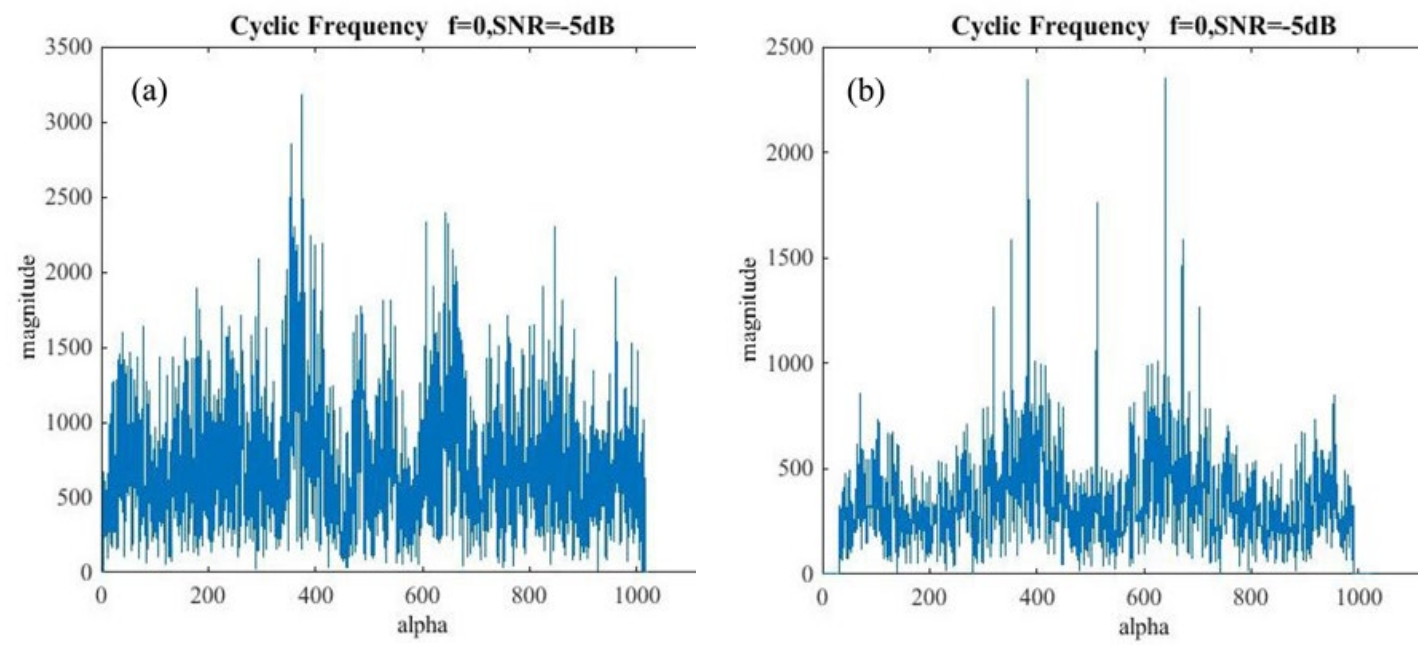

Figure 10. $\mathrm{SNR}=-5 \mathrm{~dB}$. The cyclic frequency $\alpha$ calculated by (a) SSCA and (b) recursive FSM.

Under the same SNR level, the peaks of recursive FSM in Figure 8-10 are more obvious. Especially at low SNR, the cyclic frequency of SSCA is blurred, while that of recursive FSM is still clear. That means it is easier to estimate symbol rate by using recursive FSM. This is because FSM is implemented directly in frequency domain, and there are no calculating errors in frequency-domain due to windowing.

We believe that recursive FSM can reduce the computational cost and has a good performance compared to the traditional algorithms, and this algorithm can be used in some occasions with strict

213 computing resources, such as satellite load, embedded system, portable instrument, and et al. However, 214 we concentrate on only the computation of the cyclic frequency, and have simulations at limited SNR 215 levels. 
The definition of cyclic spectrum density is introduced briefly and some different estimation

218 methods are given in this paper. On the base of cyclic spectrum density estimation method analysis, an improved frequency smoothing method using iterative process to reduce the computational cost is proposed. In addition, by comparing the simulation results of SSCA and recursive FSM at the different

221 SNR levels, recursive FSM has a good performance to acquire cyclic frequency which can be used in 222 signal recognition.

\section{Abbreviations}

224 FAM: Fast fourier transform Accumulation Method; SSCA: Strip Spectral Correlation Algorithm; FSM: Frequency Smoothing Method; GFDM: Generalized Frequency Division Multiplexing; SNR: Signal to Noise Ratio; FFT: Fast

226 Fourier Transform; BPSK: Binary Phase Shift Keying.

\section{Ethics approval and consent to participate}

228 Not applicable.

\section{Acknowledgements}

230 Not applicable.

\section{Authors' contributions}

232 Conceptualization: X.Z.F. and J.Z.D.; Methodology: X.Z.F., J.Z.D., J.Y.C., S.H.Y. and A.A.J.; Writing - original 233 draft: X.Z.F.; Writing - review \& editing: X.Z.F., J.Z.D., J.Y.C., S.H.Y. and A.A.J.

\section{Funding}

235 This research received no external funding.

\section{Availability of data and materials}

237 Data sharing not applicable to this article as no datasets were generated or analysed during the current study.

\section{Consent for publication}

239 Not applicable.

\section{Competing Interests}

241 The authors declare that they have no competing interests.

\section{Author details}

243 College of Intelligent Science and Technology, National University of Defense Technology,

244 Changsha 410073, China; 
246 yanshuhua996@163.com (S.H.Y.); jiaaiai1988@163.com (A.A.J.);

247 * Correspondence: kd805fxz@163.com; Tel.: +86-137-5516-7689.

\section{References}

249 [1] W. A. Gardner, "Spectral correlation of modulated signals: part I-analog modulation,” IEEE Transactions on Communications, vol. 35, no.6, pp.584-594, June1987.

251 [2] W. A. Gardner, "Spectral correlation of modulated signals: part II-digital modulation," IEEE Transactions on Communications, vol. 35, no.6, pp.595-601, June1987.

253 [3] W. A. Gardner, "Signal interception: a unifying theoretical framework for feature detection," IEEE Transactions on Communications, vol. 36, no.8, pp. 897-906, Aug. 1988.

255 [4] W. A. Gardner, "Exploitation of spectral redundancy in cyclostationary signals," IEEE Signal Processing, vol.8, no.2, pp. 14-36, April 1991.

[5] W. A. Gardner, "Measurement of spectral correlation," IEEE Transactions on Acoustics, Speech and Signal Processing, vol.34, no.5, pp. 1111-1123, May 1986.

[6] R. S. Roberts, W. A. Brown and H. H. Loomis, "Computationally efficient algorithms for cyclic spectral analysis,” IEEE Signal Processing, vol.8, no.2, pp. 38-49, April 1991.

261 [7] W. A. Brown and H. H. Loomis, "Digital implementations of spectral correlation analyzers," IEEE Signal Processing, vol.41, no.2, pp. 703-718, April 1993. cognitive radio," International Symposium on Communications and Information Technologies, Gold Coast, Australia, pp.663-667, 2012. algorithm for cyclostationary inputs,” Signal Processing, vol.141, no.12, pp.261-272, Dec. 2017. 
270 [11] P. Borghesani and J. Antoni, "A faster algorithm for the calculation of the fast spectral correlation," 271 Mechanical Systems and Signal Processing, vol.111, no.10, pp. 113-118, Oct. 2018.

[12] J. Antoni, G. Xin and N. Hamzaoui, "Fast computation of the spectral correlation," Mechanical Systems and Signal Processing, vol.92, no.8, pp.248-277, Aug. 2017.

274 [13] M. I. M. Alfaqawia, J. Chebilb, M. H. Habaebic and D. Datla, "Wireless distributed computing for cyclostationary feature detection,” Digital Communications and Networks, vol.2, no.1, pp.47-56, Jan.

[14] B. Ramkumar, "Automatic modulation classification for cognitive radios using cyclic feature detection,"

[15] N. J. Carter, Implementation of Cyclic Spectral Analysis Methods, Naval Postgraduate School, Monterey, 2015 .

281 [16] X. D. Zhang and Z. Bao. Analysis and Processing of Non-stationary Signals, Defense Industry Press, Beijing, 2001. 
Figures

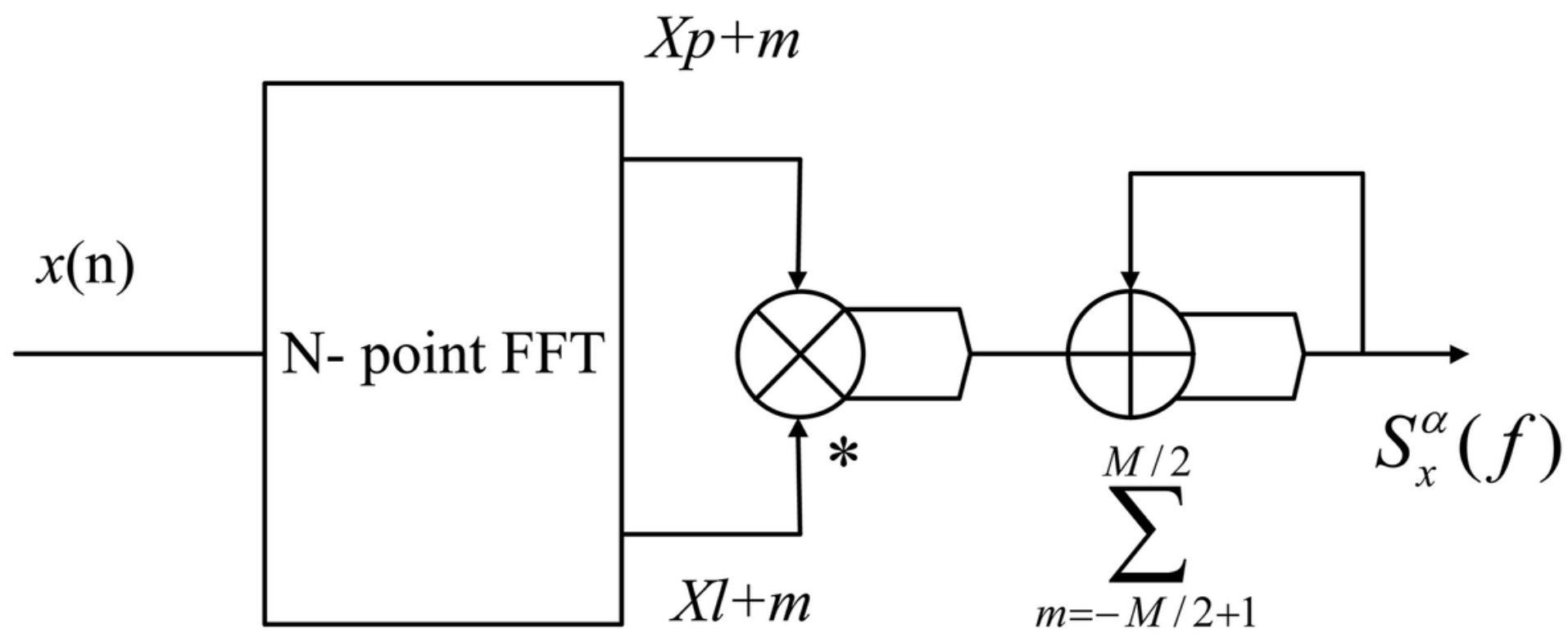

Figure 1

Frequency smoothing method architecture

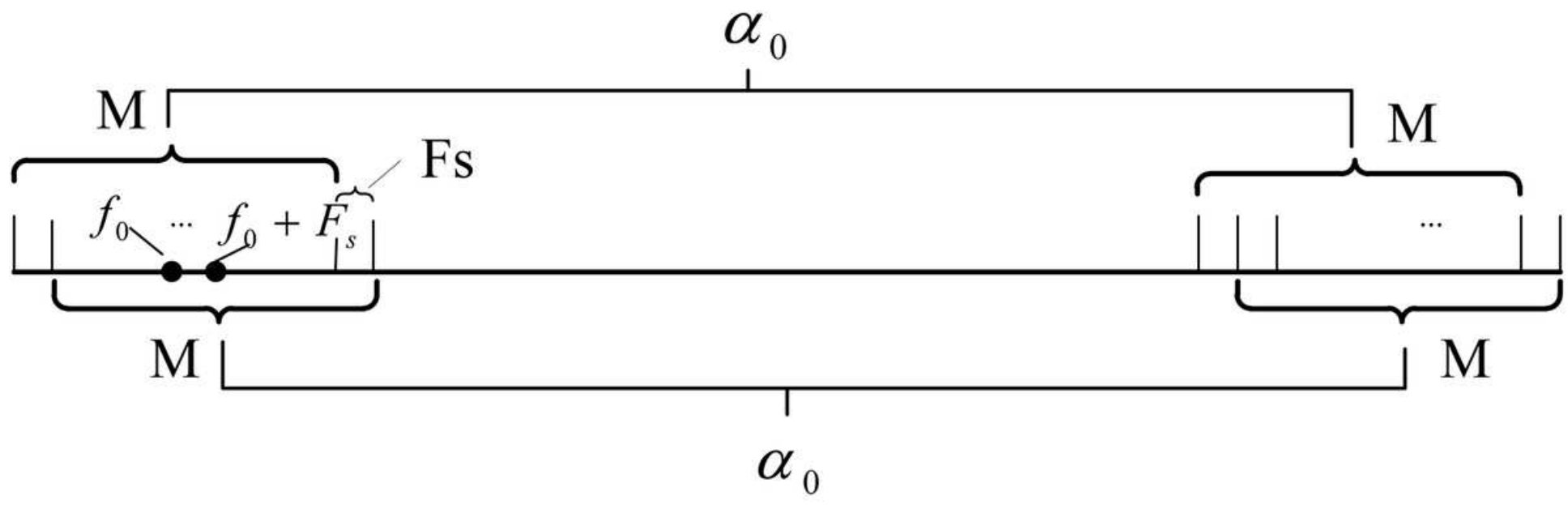

Figure 2

Sketch of smoothing window move-fixed a 


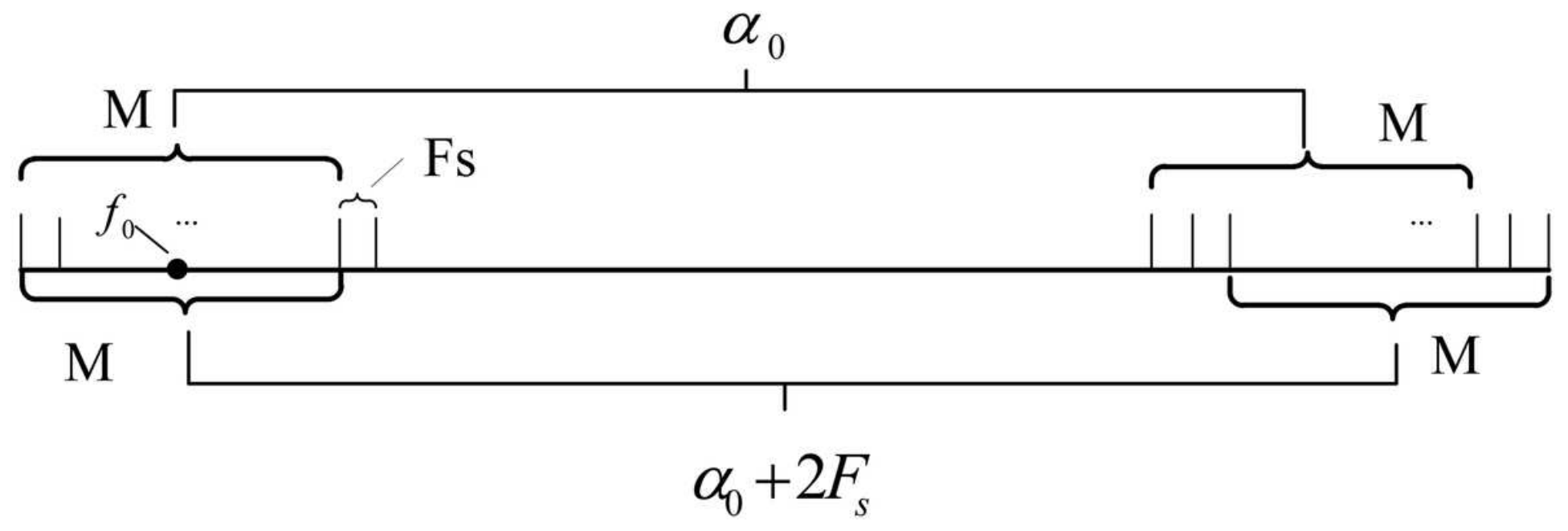

Figure 3

ketch of smoothing window move-fixed $f$

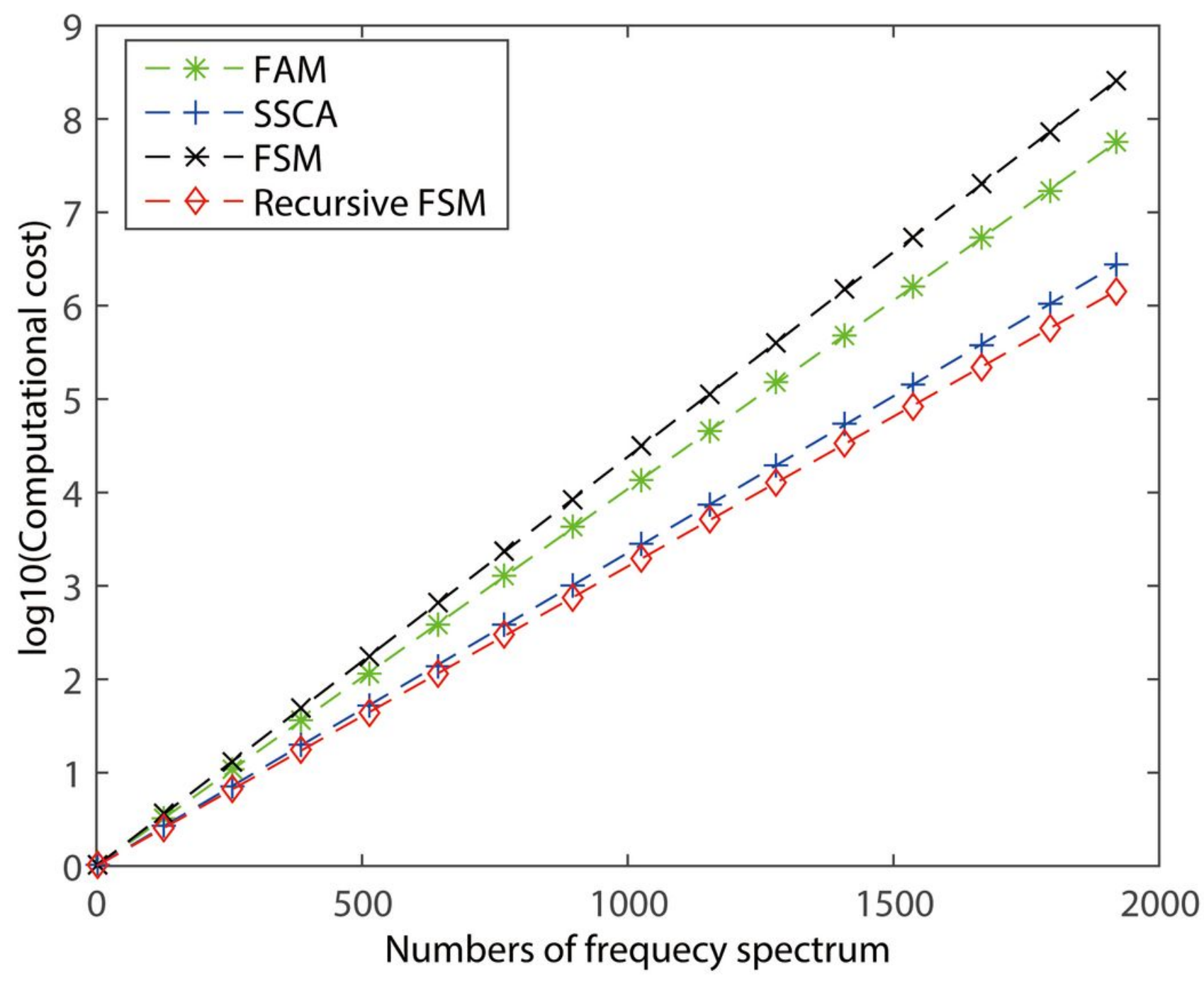

Figure 4 
Comparison of different algorithms
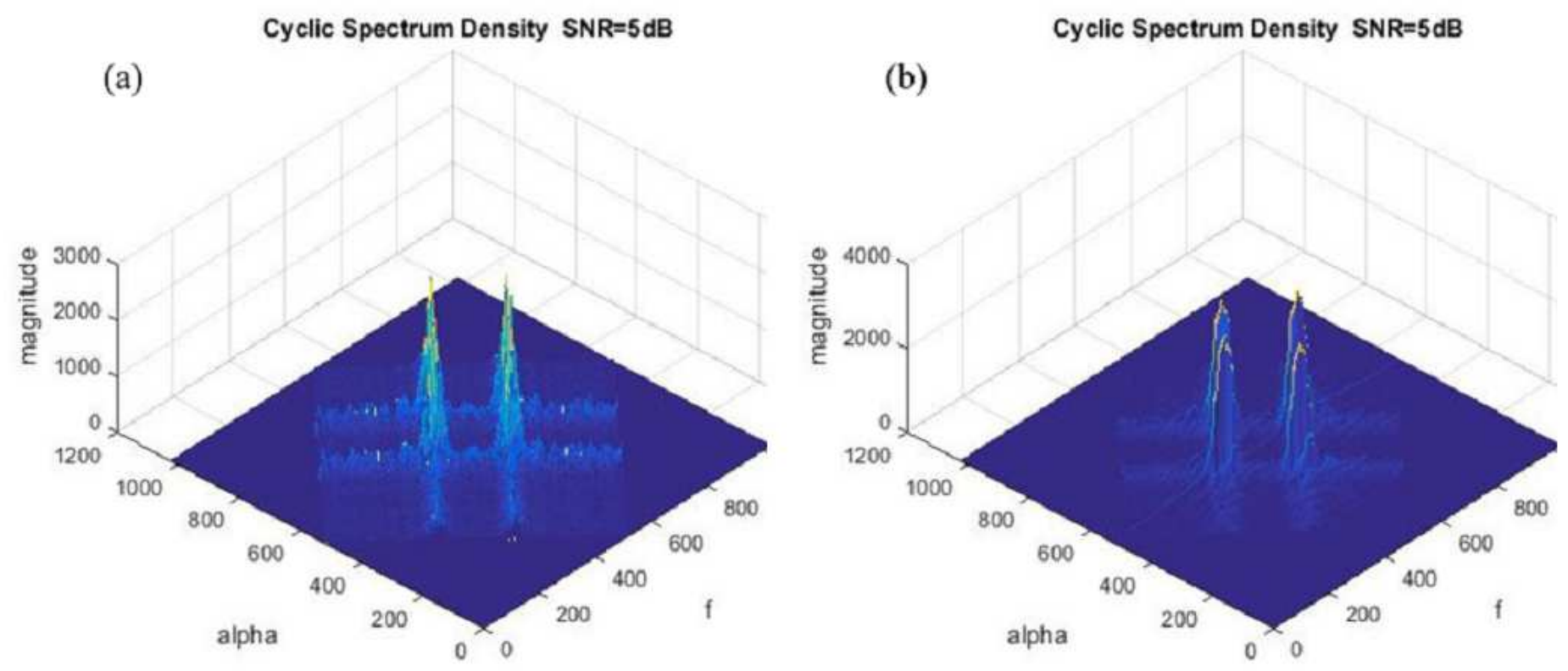

\section{Figure 5}

$S N R=5 d B$. Cyclic spectrum density calculated by (a) SSCA and (b) recursive FSM.
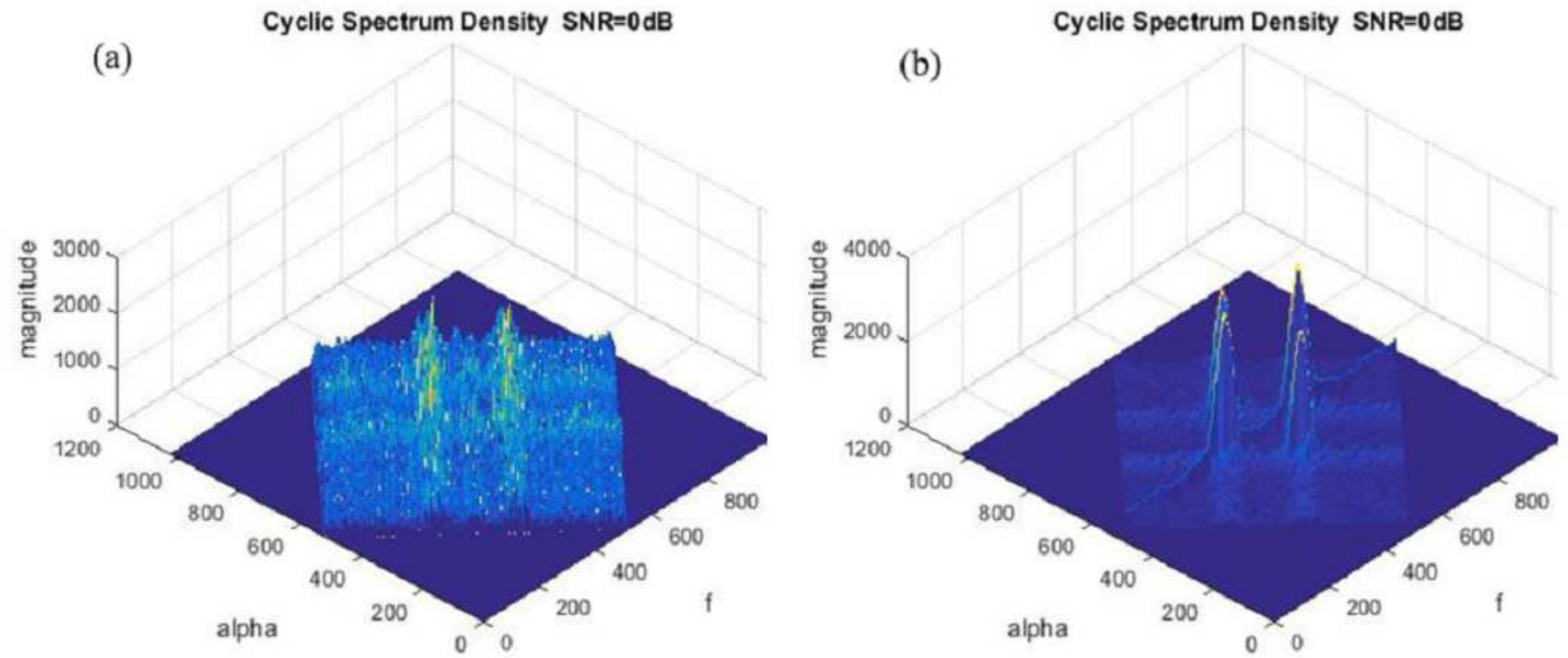

Figure 6

SNR=0dB. Cyclic spectrum density calculated by (a) SSCA and (b) recursive FSM. 

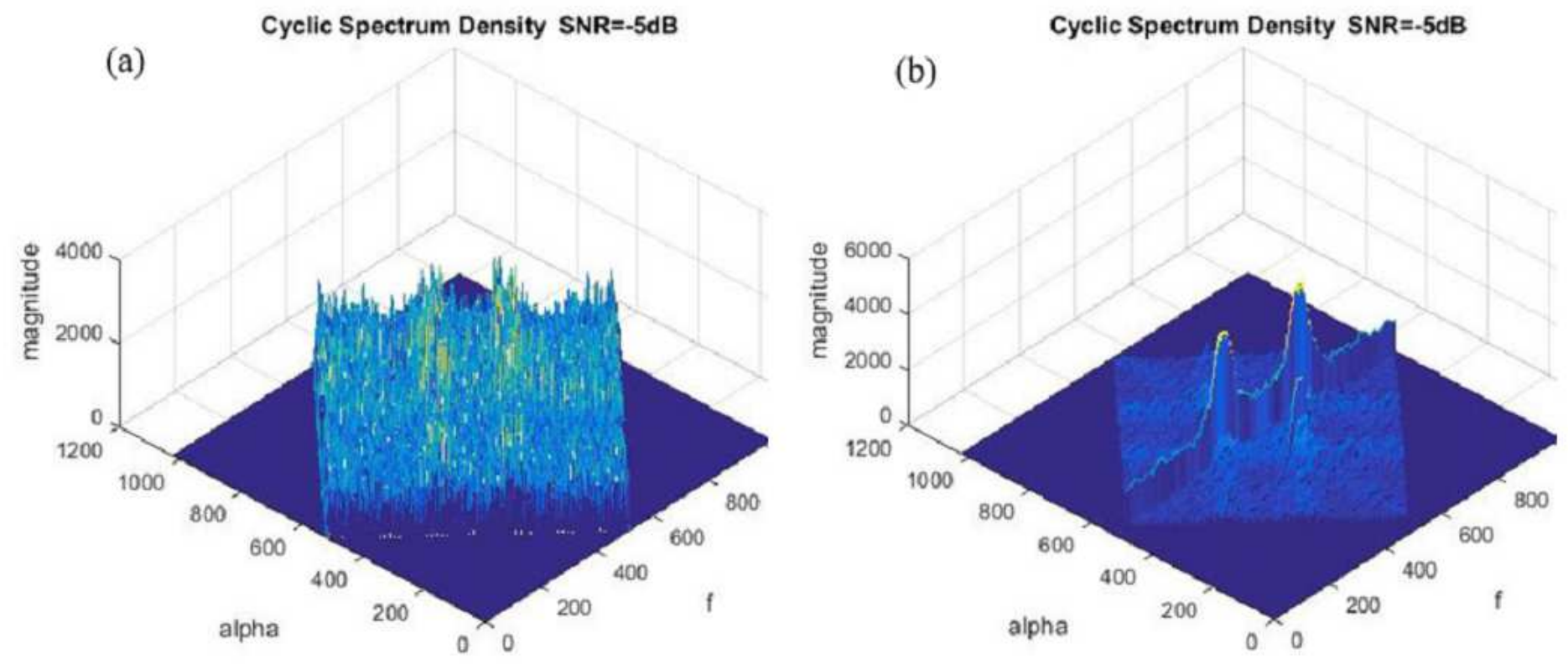

Figure 7

$S N R=-5 d B$. Cyclic spectrum density calculated by (a) SSCA and (b) recursive FSM.
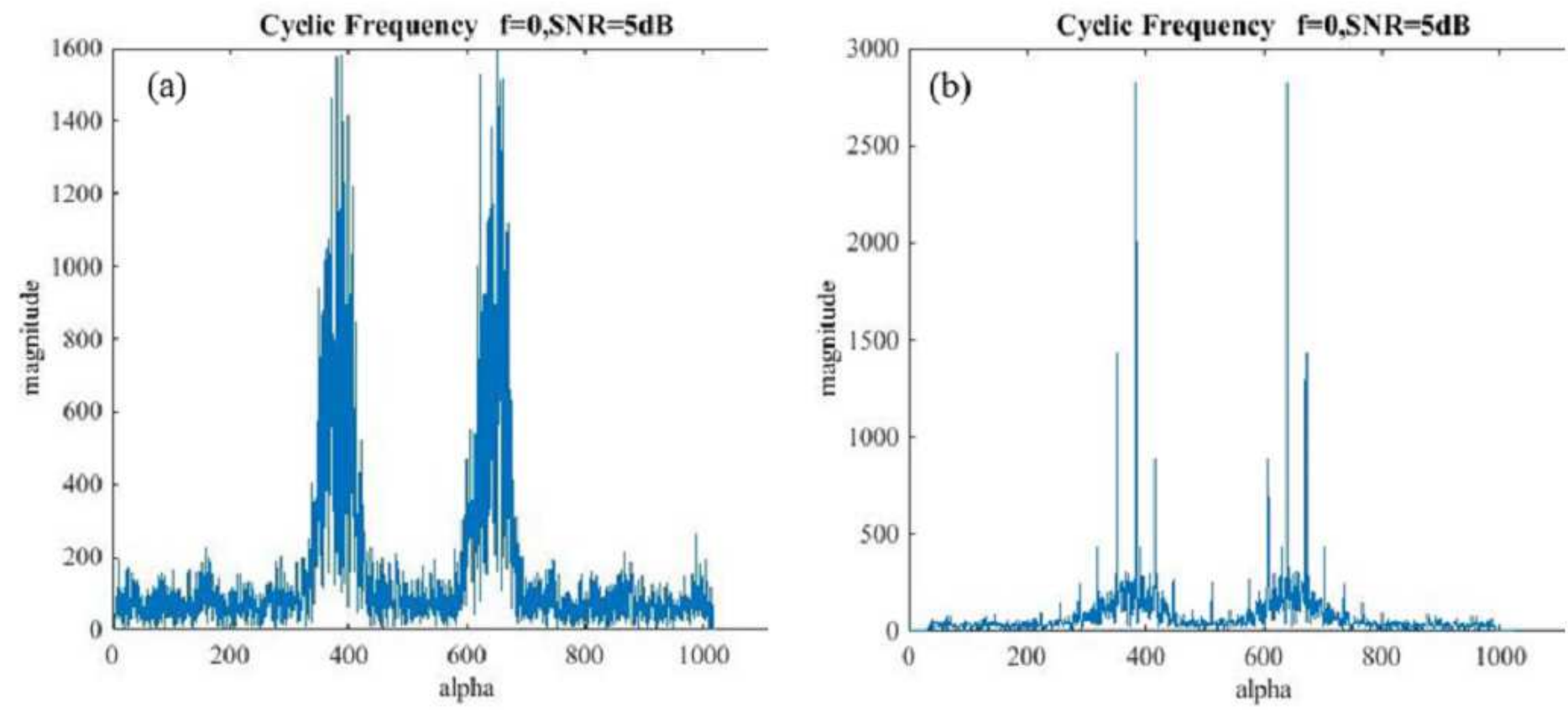

Figure 8

$S N R=5 d B$. The cyclic frequency a calculated by (a) SSCA and (b) recursive FSM. 

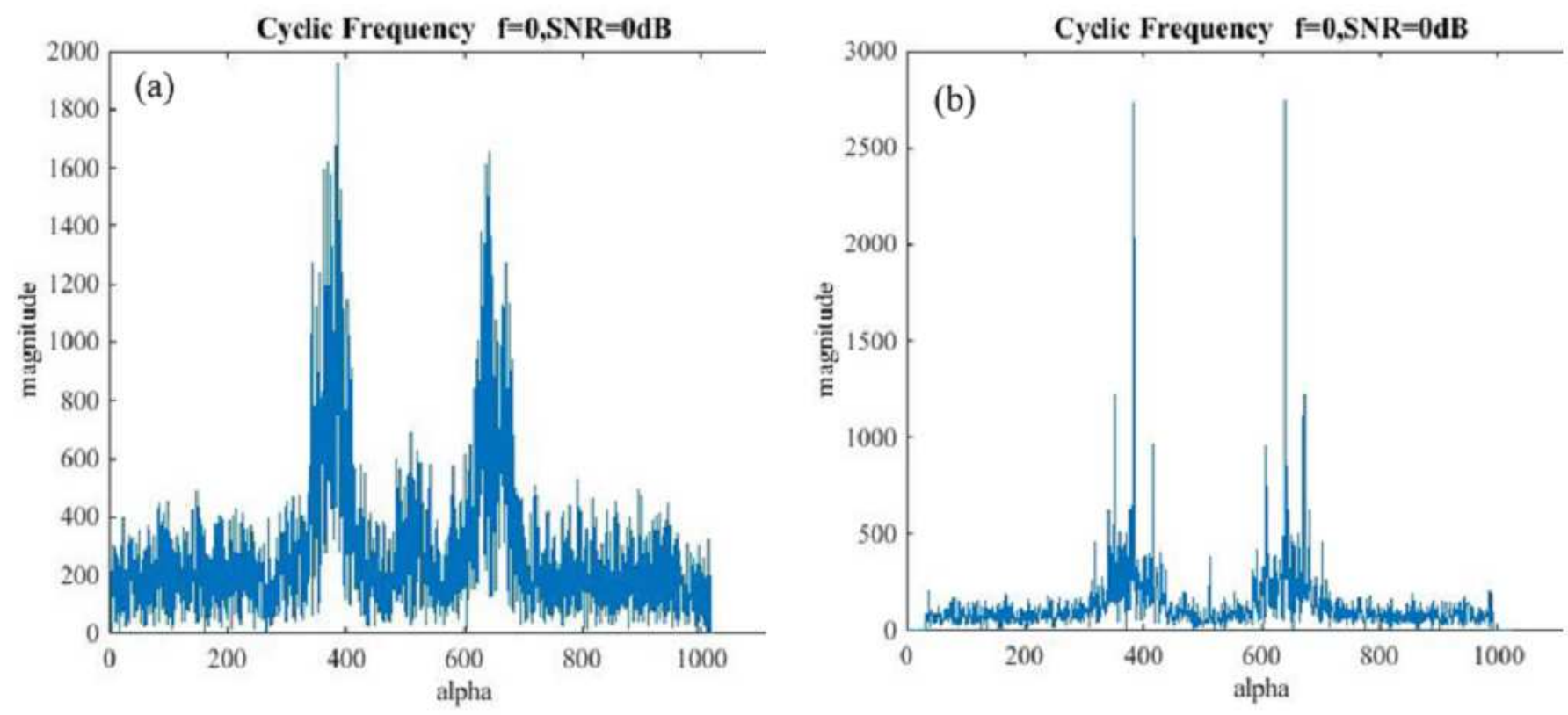

Figure 9

$S N R=0 \mathrm{~dB}$. The cyclic frequency a calculated by (a) SSCA and (b) recursive FSM.
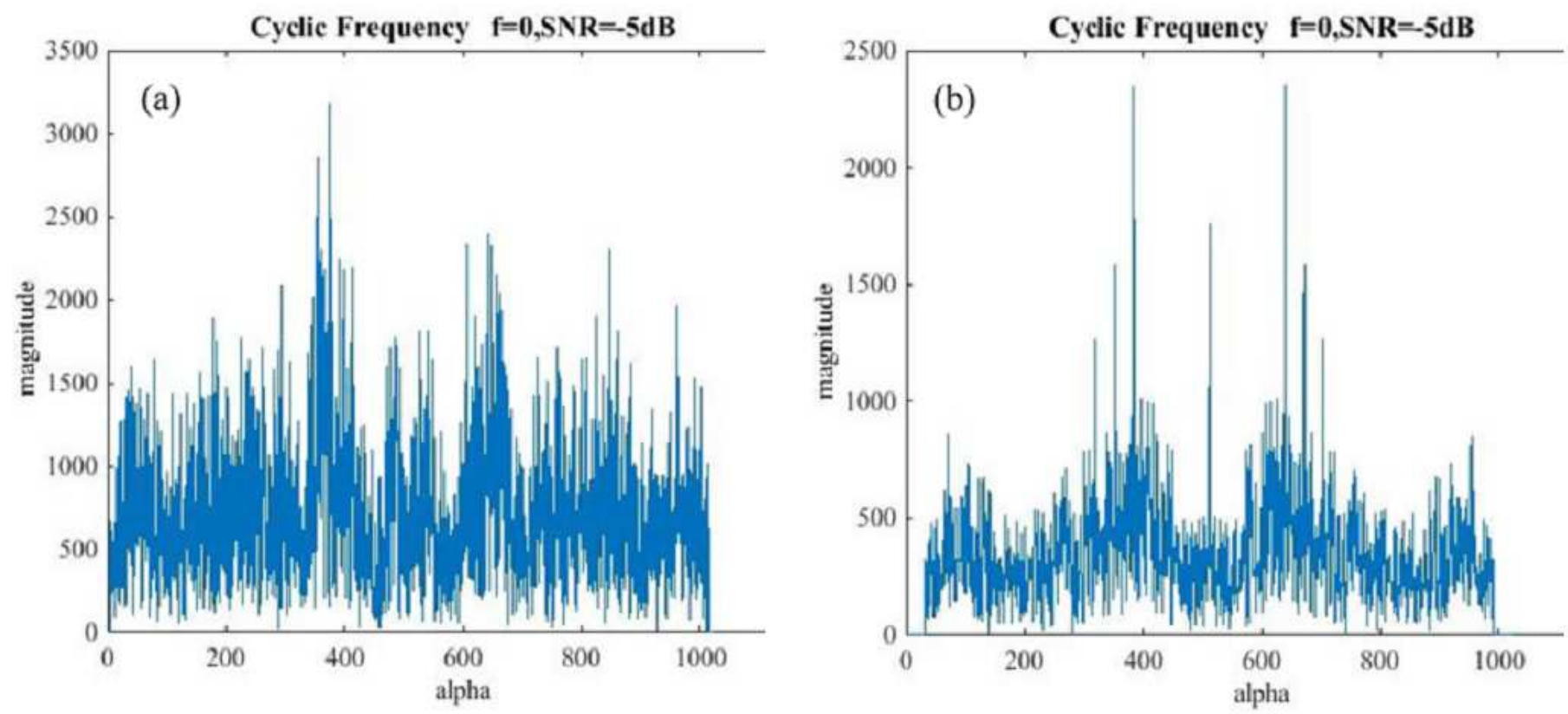

Figure 10

SNR $=-5 \mathrm{~dB}$. The cyclic frequency a calculated by (a) SSCA and (b) recursive FSM. 